\title{
Effect of captivity and cryopreservation on ROS production in Solea senegalensis spermatozoa
}

\author{
D G Valcarce ${ }^{1,2}$ and V Robles ${ }^{2,3}$ \\ ${ }^{1}$ Department of Molecular Biology, ${ }^{2}$ INDEGSAL, University of León, León, Spain and ${ }^{3}$ IEO, Spanish Institute of \\ Oceanography, Planta de Cultivos el Bocal, Barrio Corbanera, Monte, Santander, Spain
}

Correspondence should be addressed to V Robles; Email: robles.vanesa@gmail.com

\begin{abstract}
Reactive oxygen species have a great impact on spermatozoa function. Gametes from sole males born in captivity (F1) display lower quality than those from wild individuals. In this paper, the percentage of cells positive for dichlorofluorescein (DCF+) was determined by flow cytometry in wild and F1 animals, the effect of cryopreservation on DCF ${ }^{+}$cells was evaluated in both groups and the distribution of $\mathrm{H}_{2} \mathrm{O}_{2}$ within the cell was studied by confocal microscopy. Our results indicated that there are no differences in either viability or $\mathrm{DCF}^{+}$cells between wild and F1 animals when fresh samples were evaluated. However, when data were analyzed considering two different sperm populations in terms of motility, a significant decrease in viability and $\mathrm{DCF}^{+}$cells was reported in low-motile F1 spermatozoa. Cryopreservation did not alter the viability or the presence of DCF ${ }^{+}$cells in sperm samples from wild animals, but significantly decreased the viability in $\mathrm{F} 1$ samples. Distribution patterns of $\mathrm{H}_{2} \mathrm{O}_{2}$ have been established by confocal microscopy in Solea senegalensis spermatozoa: co-localization of $\mathrm{H}_{2} \mathrm{O}_{2}$ with active mitochondria (MitoTracker ${ }^{+}$) and co-localization with nuclear DNA (DAPI). Compared with $\mathrm{H}_{2} \mathrm{O}_{2}$ distribution in other marine species such as Scophthalmus maximus, Solea senegalensis spermatozoa showed widespread presence of $\mathrm{H}_{2} \mathrm{O}_{2}$ particularly in the nuclei, which could potentially compromise DNA integrity.

Reproduction (2016) 152 439-446
\end{abstract}

\section{Introduction}

Marine aquaculture producers from Southern Europe show an increasing interest in a promising species: Senegalese sole (Solea senegalensis Kaup, 1858). Sole has both a good marketable assessment and very good consumer acceptance. Despite the fact that sole is an appealing candidate for marine aquaculture, this species shows important limitations in industrial culture (Howell et al. 2006, 2009). The absence of courtship in F1 males (Porta et al. 2006) together with poor and variable semen quality, particularly in F1 males, are major obstacles in large-scale production (Cabrita et al. 2006, Martínez-Pastor et al. 2008, Beirão et al. 2009, 2011). This absence of natural reproduction in animals born in captivity makes the use of artificial fertilization methods necessary (Rasines et al. 2013). These methods involve fish manipulation for gamete collection, sperm classification by motility, and cryopreservation for storing the best sperm samples (Sieme et al. 2015) until artificial fertilization trials are performed. Only optimal samples should be used for cryopreservation so as to guarantee good in vitro fertilization results.

Reactive oxygen species (ROS) formation has been recognized as a problem for sperm survival and fertility
(Guthrie \& Welch 2012). In mammals, oxidative stress damage competence of spermatozoa by peroxidation of lipids, induction of oxidative DNA damage and formation of protein adducts are well known (Aitken et al. 2012). It has also been established that ROS production could initiate an intrinsic apoptotic cascade causing spermatozoa to lose their motility (Aitken et al. 2012). In Senegalese sole, a significantly higher percentage of apoptotic cells in F1 males was reported than those in wild-captured animals (Valcarce et al. 2016). The aim of this work was to investigate the relationship between ROS levels and sperm quality in Solea senegalensis, which presents two particular problems: (1) low sperm quality in F1 samples and (2) the need for sperm to be cryopreserved before artificial fertilization. For this purpose, the intracellular levels of ROS species and viability of sperm samples from wild-captured and F1 males were analyzed by flow cytometry in fresh and cryopreserved samples. Moreover, the distribution of $\mathrm{H}_{2} \mathrm{O}_{2}$ in the spermatozoa was studied by confocal microscopy and compared with other marine flat fish species. This analysis gave a molecular insight into sole spermatozoa providing new keys for understanding reproduction failure in Solea senegalensis. 


\section{Materials and methods}

\section{Ethics}

Animal manipulation was carried out following the national and institutional bioethical guidelines and European Union Directive 2010/63/EU for the protection of animals used for experimental and other scientific purposes. The experiments performed in this study are part of project AGL2015 68330C2$1 \mathrm{R}$ approved by the IEO ethics committee (1/2016).

\section{Animal maintenance and experimental groups}

Wild-captured (WT) and F1 broodstocks (F1) were used in this experiment. Fish labeled as wild-captured males (WT) refers to those born in their natural environment and able to reproduce naturally, whereas those labeled F1 are born in captivity and are unable to reproduce. WT males were captured two years before starting this experiment and, according to their size and weight, were approximately 4 years old. F1 males were 4 years old. Both groups of animals were held indoors at a 1:1 female:male ratio in tanks $\left(4-14 \mathrm{~m}^{2}\right.$ in area and $1 \mathrm{~m}$ deep) with a stock density around $5 \mathrm{~kg} / \mathrm{m}^{3}$ in the Spanish Institute of Oceanography in Santander (Marine Culture Plant El Bocal). Each tank maintained constant aeration and an open flow circuit $(33 \%$ tank/h of water renewal). They were exposed to a $16: 8 \mathrm{~h}$ (light:darkness) continual artificial photoperiod. Light intensity was reduced with mesh shading placed over the tanks. The fish were fed $(0.5 \%$ of the total biomass) 6 days a week with commercially extruded feed (Vitalis Cal and Vitalis Repro, Skretting). Each animal in the culture plant was monitored with passive integrated transponder tags (PIT tags, AVID) inserted in the dorsal musculature. Three WT $(n=3)$ and six F1 $(n=6)$ fish were used for this experiment.

\section{Sperm sample collection}

Before collection, the males were anesthetized with $40 \mathrm{ppm}$ clove oil for $2 \mathrm{~min}$. The urogenital pore was always toweled to remove feces, water and mucus before sperm sampling. Contaminated sperm was rejected. Ejaculates were collected with a syringe without a needle by gently pressing the testes on the pigmented side of the fish. The samples were individually maintained at $4{ }^{\circ} \mathrm{C}$ in microcentrifuge tubes until processing.

\section{Sperm analysis}

For each ejaculate (WT: $n=3$ and F1: $n=6$ ), cell concentration and volume were analyzed. Percentage of motile spermatozoa was assessed using the scores by Billard and coworkers (1995). For this purpose, $1 \mu \mathrm{L}$ of prediluted sperm (1:10) in $200 \mathrm{mOsm} / \mathrm{kg}$ Ringer solution $(116 \mathrm{mM} \mathrm{NaCl} ; 2.9 \mathrm{mM} \mathrm{KCl}$; $1.8 \mathrm{mM} \mathrm{CaCl}_{2} ; 5 \mathrm{mM}$ HEPES, $\left.\mathrm{pH} 7.7\right)$ was activated with $500 \mu \mathrm{L}$ artificial seawater $(24.6 \mathrm{~g} / \mathrm{L} \mathrm{NaCl}, 0.67 \mathrm{~g} / \mathrm{L} \mathrm{KCl}, 1.36 \mathrm{~g} / \mathrm{L}$ $\mathrm{CaCl}_{2} \cdot 2 \mathrm{H}_{2} \mathrm{O}, 6.29 \mathrm{~g} / \mathrm{L} \mathrm{MgSO}{ }_{4} \cdot 7 \mathrm{H}_{2} \mathrm{O}, 4.66 \mathrm{~g} / \mathrm{L} \mathrm{MgCl} \cdot 6 \mathrm{H}_{2} \mathrm{O}$, $0.18 \mathrm{~g} / \mathrm{L} \mathrm{NaHCO}_{3} ; 1100 \mathrm{mOsm} / \mathrm{kg} ; \mathrm{pH} 8.0$ ) kept at $4{ }^{\circ} \mathrm{C}$. Motility was determined under light contrast microscopy (magnification: 200x). This procedure was performed twice for each sperm sample following the method described by Billard (Billard et al. 1995).

\section{Cryopreservation protocol}

Each sample was split into two: (1) a fresh aliquot (FRESH) and (2) a cryopreserved aliquot (CRYO). For cryopreservation, cells were diluted (1:2 ratio) in Mounib extender with cryoprotectants (10\% BSA and 10\% DMSO) following the protocol published by Rasines (Rasines et al. 2013). The samples were loaded into $0.5 \mathrm{~mL}$ straws and exposed to liquid nitrogen vapor for $7 \mathrm{~min}$. After this time, the straws were immersed in liquid nitrogen.

\section{Thawing protocol}

The samples were thawed in a $40^{\circ} \mathrm{C}$ bath for $7 \mathrm{~s}$. The cryoprotectants were discarded after centrifugation (1 min; $1000 \mathrm{~g}$ ) and the cells were resuspended in Ringer solution.

\section{Flow cytometry analysis}

Analyses were performed using a NovoCyte Flow Cytometer (Acea Biosciences, San Diego, CA, USA). Viability was measured using propidium iodide (PI) (Sigma), and intracellular sperm ROS levels were studied using dichlorofluorescein diacetate (DCFH-DA) (Sigma), which can precisely reveal intracellular ROS, emitting green fluorescence when oxidized (Fig. 1). Both aliquots (FRESH and CRYO) for each individual (WT and F1) were incubated in $25 \mu \mathrm{M} \mathrm{DCFH-DA}\left(7^{\circ} \mathrm{C}, 40 \mathrm{~min}\right.$ ) and co-stained with $2 \mu \mathrm{g} / \mathrm{mL} \mathrm{PI}$ (Invitrogen) $\left(7^{\circ} \mathrm{C}, 10 \mathrm{~min}\right)$. Overall, 10,000 events were acquired per sample, and the acquisition was performed twice for each sample. Data analysis was performed using NovoExpress software (Acea Biosciences).
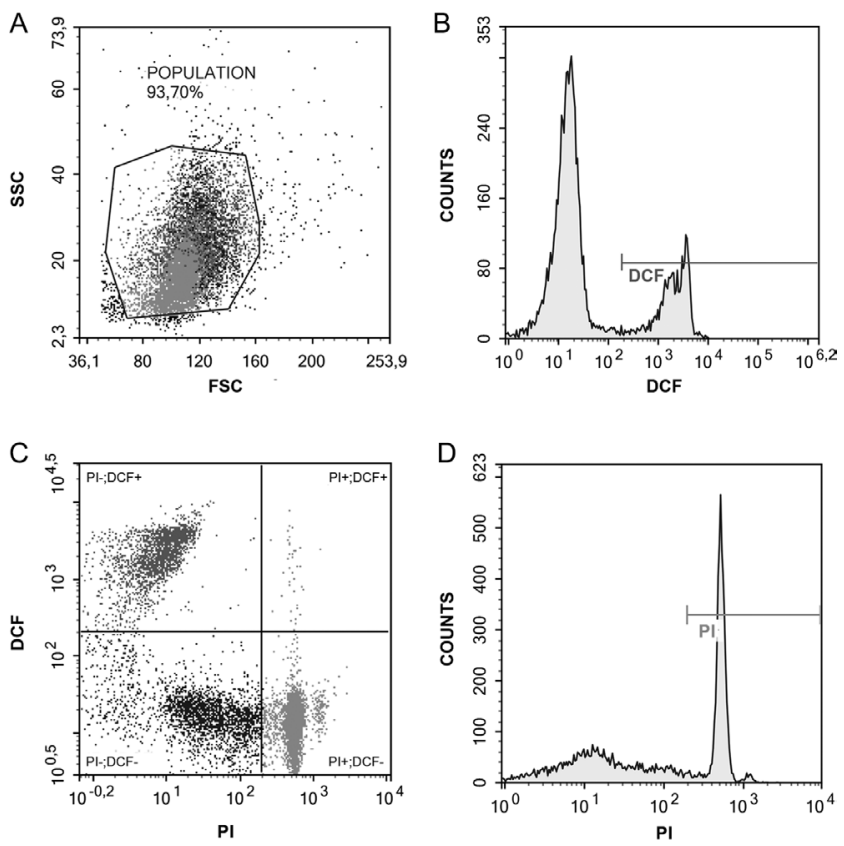

Figure 1 Flow cytometry examples. (A) Dot plot cytograms showing population gating (SSC vs FSC), (B) simultaneous measurements of intracellular reactive oxygen species (DCF) and dead cells (PI). Single count histograms for (C) DCF and (D) PI. DCF, dichlorofluorescein; FSC, forward scatter; PI, propidium iodide; SSC, size scatter. 
As control of the technique for the detection of ROS with DCFH-DA in Solea senegalensis sperm, three samples were split into three: (i) a non-treated aliquot, (ii) an aliquot treated with $100 \mathrm{mM}$ sodium pyruvate (Sigma-Aldrich), a scavenger of reactive oxygen species and (iii) an aliquot treated with $10 \%$ $\mathrm{H}_{2} \mathrm{O}_{2}$ (Sigma-Aldrich) for $15 \mathrm{~min}$ as a positive control. Controls were washed in $1 \times$ PBS after treatment and subsequently processed in the usual way.

\section{Measurement of reduced glutathione}

To analyze the antioxidant activity in WT and F1 spermatozoa, we studied the intracellular reduced glutathione content for both wild-captured $(n=3)$ and F1 spermatozoa cryopreserved samples $(n=3)$. For this purpose, we used a Glutathione Colorimetric Assay Kit (Biovision). In brief, $1 \times 10^{6}$ cells from wild-captured and F1 sperm respectively were used as samples. After lysis and centrifugation, the supernatants were used for the reduced glutathione measurement in a microplate reader as per the manufacturer's protocol.

\section{Confocal microscopy analysis}

To localize the presence of intracellular ROS in Senegalese sole sperm, $1-2 \times 10^{6}$ cells $/ \mathrm{mL}$ of WT, F1 and controls (15 min; $10 \mathrm{mM}$ sodium pyruvate and $15 \mathrm{~min} 10 \%$ hydrogen peroxide) were incubated with $25 \mu \mathrm{M}$ DCFH-DA (Sigma) $\left(7^{\circ} \mathrm{C}, 40 \mathrm{~min}\right)$ and $100 \mathrm{nM}$ MitoTracker Deep Red (Invitrogen) $\left(7^{\circ} \mathrm{C}, 10 \mathrm{~min}\right)$. Nuclei were labeled with 4',6-diamidino-2-phenylindole (DAPI) (Sigma-Aldrich; 1:1000). A $5 \mu \mathrm{L}$ cell suspension drop was placed on a slide and immediately analyzed under a LSM 800 confocal microscope (Zeiss). To compare the localization of intracellular $\mathrm{H}_{2} \mathrm{O}_{2}$ in other flatfish species, confocal microscopy analysis was used following the same protocol described previously with turbot (Scophthalmus maximus) sperm cells.

\section{Statistical methods}

Data were analyzed using SPSS, version 20 for Macintosh (SPSS). Data are presented as mean \pm S.E.M. in all cases. Mean values of each variable were compared by a one-way ANOVA $(P<0.05)$. Duncan post hoc test was performed to analyze homogenous subgroups in each parameter.

\section{Results}

\section{Sperm viability}

Wild-captured vs F1 males

No statistical differences were found between wildcaptured (WT-FRESH) and F1 (F1-FRESH) males in fresh samples (Fig. 2 - full colored bars). Good viability (over $60 \%$ in both cases) was reported in fresh samples.

\section{Fresh vs cryopreserved samples}

Two different patterns were shown: cryopreservation did not significantly decrease viability in wild-captured males (WT-CRYO) (only a reduction of less than 5\% of

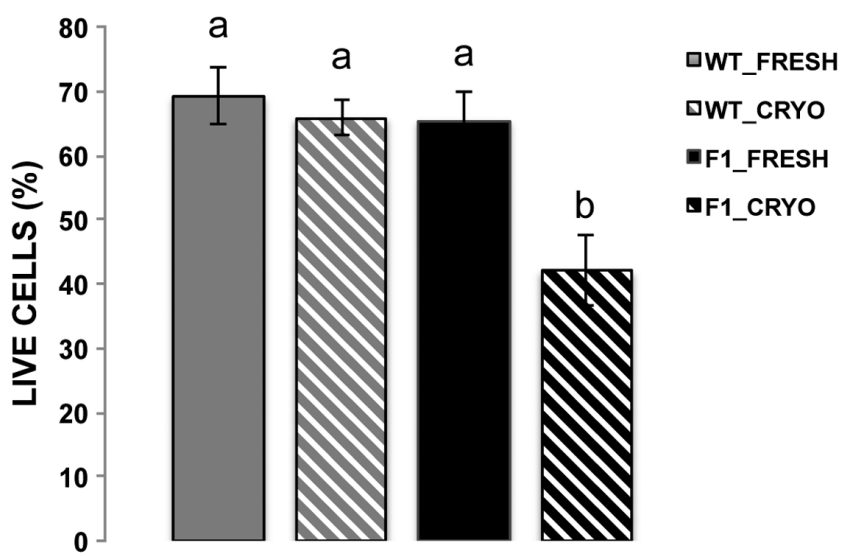

Figure 2 Viability analysis by flow cytometry: WT $(n=3)$ vs F1 $(n=6)$. CRYO, cryopreserved/thawed samples; F1, F1 males; FRESH, control samples before cryopreservation; WT, wild-captured males. Superscript letters show differences among groups $(P<0.05)$.

viable cells was recorded) (Fig. 2). On the other hand, males born in captivity (F1-CRYO) were strongly affected by the protocol reporting a statistically significant reduction of around $35 \%$.

F1 generation: high-motility samples vs low-motility samples

To check if the best F1 samples in terms of motility showed a similar tendency to wild male samples, data were reanalyzed in F1 attending to motility. Wildcaptured male samples were kept as reference. The F1 group was split into two groups according to their motility (Supplementary Material 1, see section on supplementary data given at the end of this article). Three of them were labeled 'low-motility samples' (F1-L MOT; $n=3$ ) with a percentage of motile cells $\leq 30 \%$, and another three were considered high-motility samples (F1-H MOT; $n=3$ ) with motility values $\geq 45 \%$ ). In F1 fresh high-motility samples (Supplementary Material 1A; F1-FRESH-H MOT), viability was similar to sperm samples from wild animals (WT-FRESH), but in low-motility samples (Supplementary Material 1B; F1-FRESH-L MOT), a significant reduction in viability was observed in F1 fresh spermatozoa (54.66 $\pm 5.41 \%$ ). After cryopreservation, sperm viability in F1 males decreased in both high- (F1-CRYO-H MOT) and lowmotility samples (F1-CRYO-L MOT) (high motility: $56.71 \pm 3.94 \%$ and low motility: $27.39 \pm 2.44 \%$ ) (Supplementary Material 1A and B - black striped bars).

\section{Sperm ROS level by flow cytometry}

The evaluation of intracellular ROS species in Solea senegalensis spermatozoa with DCF by flow cytometry was corroborated by an independent experiment (Supplementary Material 2). Samples treated with sodium pyruvate (scavenger of ROS) presented significantly fewer DCF $^{+}$cells, whereas those treated with $\mathrm{H}_{2} \mathrm{O}_{2}$ reported higher values than the non-treated sample. 
Wild-captured vs F1 males

WT fresh samples (WT-FRESH) presented high levels of $\mathrm{DCF}^{+}$cells over $50 \%$. There were no statistical differences between this group and F1 fresh samples (F1-FRESH) although a clear tendency of reduction can be seen in the results (Fig. 3 - full colored bars).

Fresh vs cryopreserved samples

No significant differences were found between fresh (WT-FRESH) and cryopreserved samples (WT-CRYO) in wild-captured males, although lower levels of $\mathrm{DCF}^{+}$cells were found in cryopreserved samples (WT-FRESH: $53.32 \pm 4.89 \%$ and WT-CRYO: $40.10 \pm 5.40$ ) (Fig. 3 - gray bars). The F1 males were not significantly affected by the freezing-thawing process. F1 fresh samples (F1-FRESH) reported $34.76 \pm 6.61 \% \mathrm{DCF}^{+}$cells (mean value \pm S.E.M.) and cryopreserved spermatozoa showed $21.72 \pm 5.81 \%$ (mean values \pm S.E.M.) (Fig. 3 - black bars).

\section{F1 generation: high-motility samples vs low-motility samples}

The previously described split was carried out in this analysis for the F1 generation: high-motility samples (F1-H MOT) vs low-motility samples (F1-L MOT).

High-motility samples reported the same tendency as the global analysis (Supplementary Material $1 \mathrm{C}$ and D). Statistical differences were only found between WT-FRESH and F1-CRYO-H MOT samples (Supplementary Material 1C).

F1 low-motility samples (F1-L MOT) presented a different profile (Supplementary Material 1D) compared with high-motility ones. These samples reported a reduction of $66 \% \mathrm{ROS}^{+}$cells compared with WT fresh samples. Following the same tendency, F1 low-motility cryopreserved samples (F1-CRYO-L MOT) showed a reduction of around $84 \%$ compared

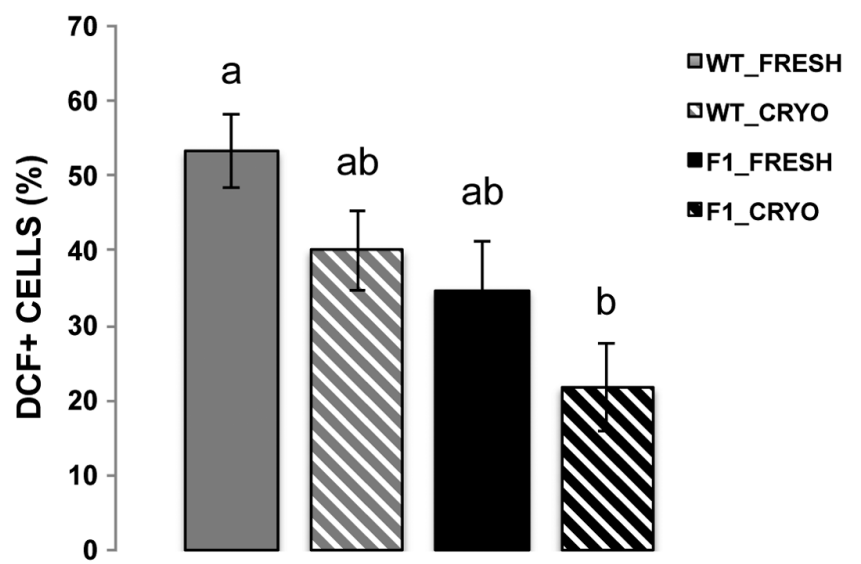

Figure 3 Intracellular reactive oxygen species analysis $\left(\mathrm{DCF}^{+}\right.$cells) by flow cytometry. WT $(n=3)$ vs F1 $(n=6)$. CRYO, cryopreserved/thawed samples; DCF, dichlorofluorescein; F1, F1 males; FRESH, control samples before cryopreservation; WT, wild-captured males. Superscript letters show differences among groups $(P<0.05)$. with the WT-CRYO group. No statistical differences were found between fresh and cryopreserved in F1 low-motility samples.

\section{Reduced glutathione levels by colorimetric assay}

The colorimetric assay reported a statistically significant higher level of reduced glutathione in F1 samples compared with that in wild-captured ones (Fig. 4).

\section{Cell localization of intracellular $\mathrm{H}_{2} \mathrm{O}_{2}$}

Confocal microscopy images showed a co-localization of ROS (green fluorescence, DCF labeling) with mitochondria (red fluorescence, MitoTraker labeling) (Fig. 5). The mitochondrial ring is located at the base of the heads of Solea senegalensis spermatozoa, which is observed as intense fluorescent areas in the confocal captures (Fig. 5D). $\mathrm{H}_{2} \mathrm{O}_{2}$ is also detected in nuclei (blue, DAPI labeling) showing that oxidative stress is not only present in mitochondria but also could be damaging DNA. This localization pattern was reported in WT and $\mathrm{F} 1$ samples (Fig. $5 \mathrm{H}$ and I). The intensity of the DCF labeling in the nuclei of Solea senegalensis spermatozoa pointed to a larger presence of ROS species in this compartment of the spermatozoa from Solea senegalensis than in other flat fish such as Scophthalmus maximus (Supplementary Materials 3 and 4).

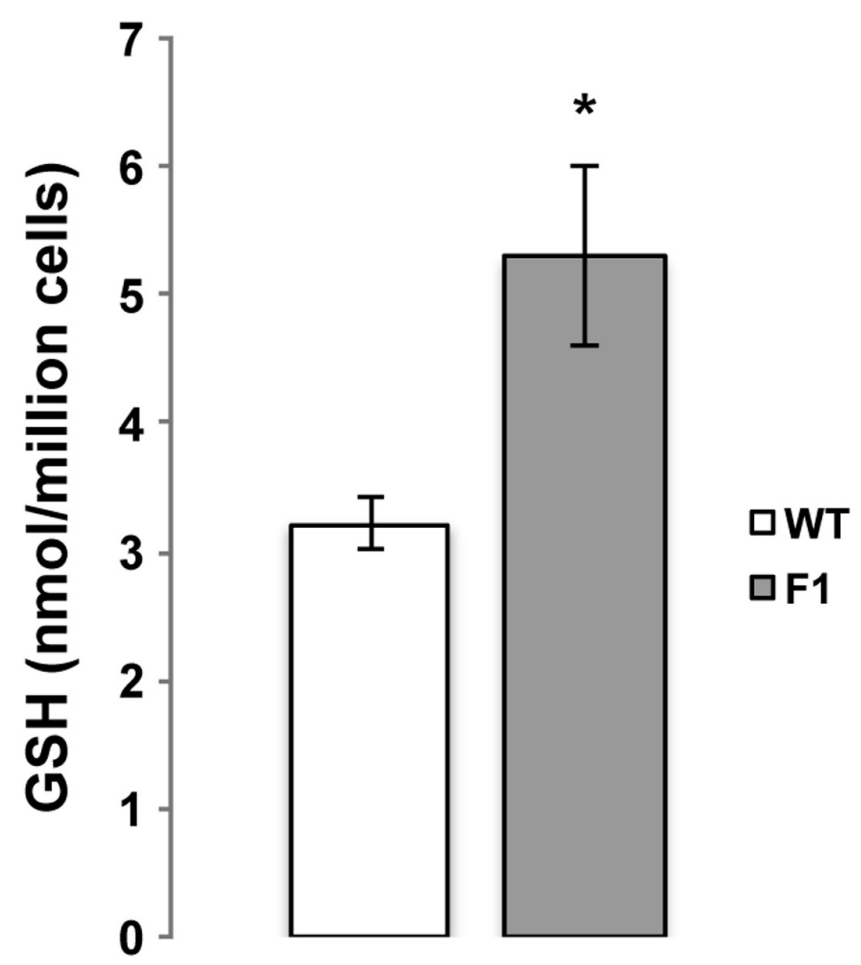

Figure 4 Reduced glutathione levels by colorimetric assay. WT $(n=3)$ vs F1 $(n=3)$. F1, F1 males; WT, wild-captured males. Asterisk shows statistically significant differences $(P<0.05)$. 

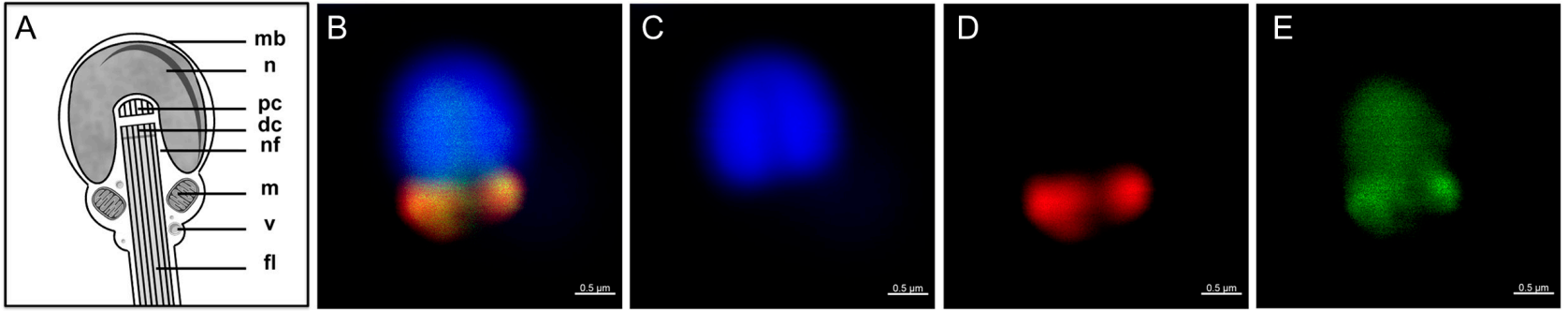

$\mathrm{F}$
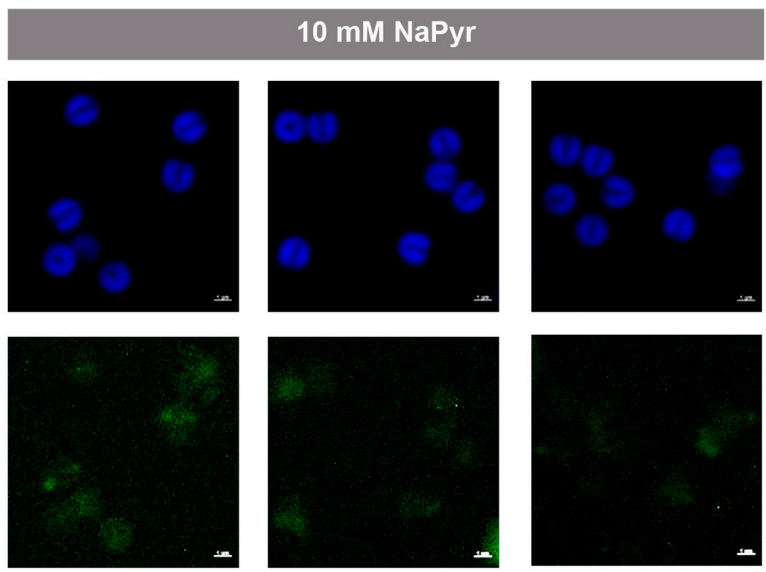

$\mathrm{H}$
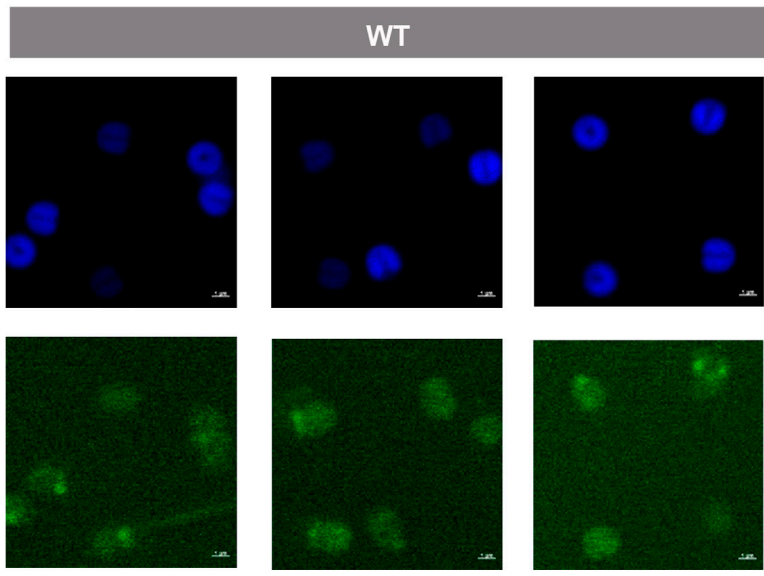

G
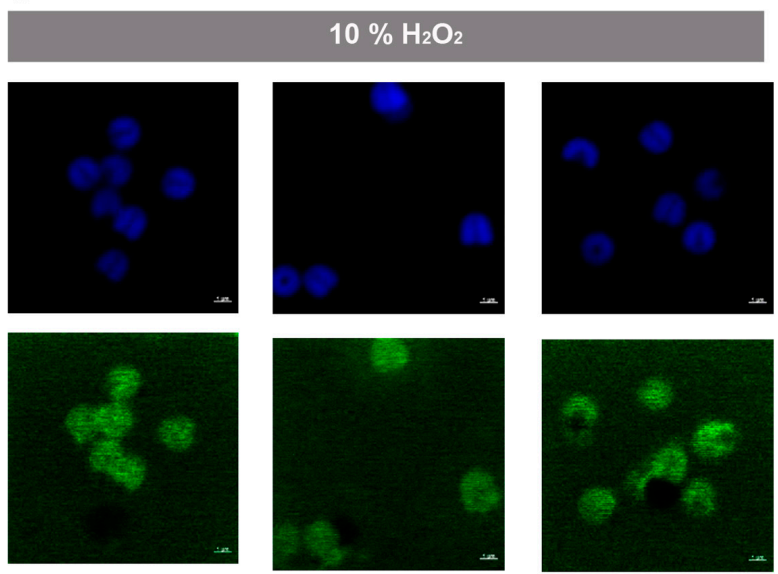

I

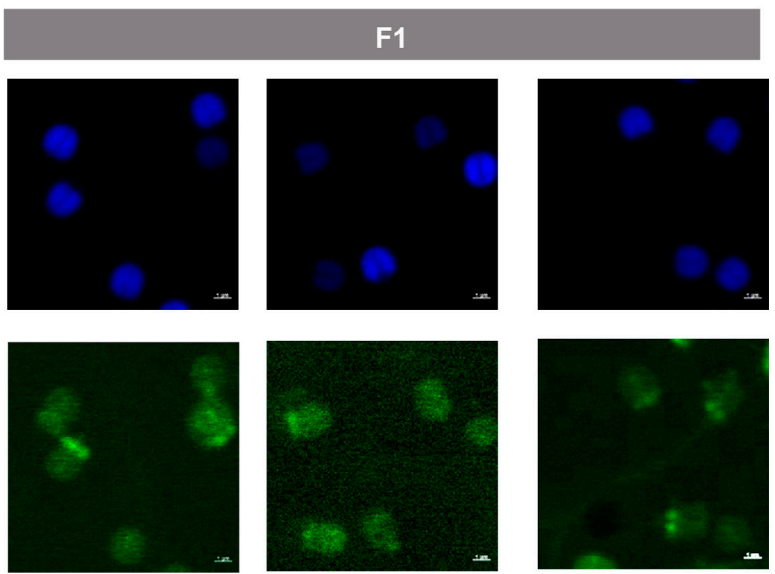

Figure 5 Intracellular reactive oxygen species localization by confocal microscopy. (A) Solea senegalensis sperm diagram. mb, membrane; $\mathrm{n}$, nucleus; pc, proximal centriole; dc, distal centriole; $\mathrm{nf}$, nuclear fossa; $\mathrm{m}$, mitochondria; v, vesicles; fl, flagellum. (B) merge (C) DNA labeling (DAPI). (D) Mitochondria labeling (MitoTracker Deep Red). (E) Intracellular ROS labeling (DCF). Examples of fields (upper fields: DAPI; lower fields: DCF) for (F) $10 \mathrm{mM} \mathrm{NaPyr}$ (ROS scavenger); (G) 10\% $\mathrm{H}_{2} \mathrm{O}_{2}$ (positive control) (H) WT and (I) F1. DAPI, 4', 6-diamidino-2-phenylindole; DCF, dichlorofluorescein; F1, F1 males; NaPyr, Sodium pyruvate; WT, wild-captured males.

\section{Discussion}

Reactive oxygen species (ROS) have usually been associated to defective sperm function causing cellular damage at different levels. The major source of ROS in spermatozoa is mitochondria. It is well known that oxidative stress produces peroxidation of lipids (Cabrita et al. 2014). It is described that mammalian spermatozoa are particularly vulnerable to this type of damage due to the high amount of polyunsaturated fatty acids (PUFA), which are very vulnerable to free radical attack (Aitken et al. 2012). Lipid peroxidation is very harmful, having a clear negative effect on sperm motility and fertilization (Aitken \& Curry 2011). ROS can even trigger an intrinsic apoptotic cascade. It is thought that the only product generated during this cascade capable of producing DNA damage is the $\mathrm{H}_{2} \mathrm{O}_{2}$ released from the mitochondria because it is a small chargeless molecule, able to penetrate the nucleus (Aitken et al. 2012). The mechanism by which DNA breakage is caused is by the oxidation of vulnerable bases, particularly guanines, which generates 8-hydroxy, 2-deoxyguanosine 
(8OHdG). All these events lead to a decrease in motility, viability and DNA integrity and therefore significantly reduce sperm quality (Aitken et al. 2012).

Our study aims to determine the effect the processes such as cryopreservation has on the levels of intracellular $\mathrm{H}_{2} \mathrm{O}_{2}$ in Solea senegalensis spermatozoa. Moreover we try to elucidate if the sperm sample's origin (from wild animals vs animals born in captivity) has an effect on the presence of ROS in these cells. Finally, we studied the distribution of this molecule in the spermatozoa.

To date, studies carried out on oxidative stress of fish could be divided into different types: (1) some reports study the ROS detoxification mechanism (Chauvigné et al. 2015), (2) others study the effect of UV irradiation or other treatments inducing oxidative stress on spermatozoa (Dietrich et al. 2005, Gazo et al. 2015), (3) other approaches evaluate the effect of the addition of antioxidant compounds or the effect of enriched diets (Beirão et al. 2015), (4) others study lipid peroxidation and its relation to decrease in sperm quality (Martínez-Páramo et al. 2012), and finally (5) others evaluate the antioxidant status of seminal plasma and spermatozoa (Shaliutina et al. 2013, Słowińska et al. 2013). Interestingly, in mammals, it has been suggested that seminal plasma could be considered as one of the most powerful antioxidant fluids (Aitken et al. 2012). The common feature of all these types of studies in fish is that ROS are considered deleterious for spermatozoa. From this perspective, our results would indicate that Solea senegalensis spermatozoa have high levels of oxidative stress $\left(\mathrm{DCF}^{+}\right.$cells, on average: $53.32 \pm 4.89 \%$ in WT and $34.76 \pm 6.60 \%$ in F1) (Fig. 3), and this could make them prone to suffering different types of damage. Surprisingly, the number of $\mathrm{DCF}^{+}$cells is significantly lower in cryopreserved F1 samples. It is well known that cryopreservation could induce oxidative stress, accelerating ROS production (Thomson et al. 2009, Kim et al. 2010, Aitken et al. 2012). So how can this fact be explained? It is known that the induction of ROS generation by sperm mitochondria is a very early stage within the whole oxidative stress process (Koppers et al. 2008, 2011), which, as expected, happens before DNA damage is produced and cell viability decreases. One possibility is that the oxidative stress process in F1 cryopreserved spermatozoa is in a later stage, so there is much more damage, but levels of ROS are not as high. In fact, when we look at viability results, we observe a significantly lower percentage of viable cells in this group $(42.05 \pm 5.61 \%$, Fig. 2$)$. In accordance with this hypothesis, when we divided the sperm samples in terms of motility for data analysis, we observed that low-motility samples have a lower percentage of $\mathrm{DCF}^{+}$ cells not only after cryopreservation but also when fresh (Supplementary Material 1). When we look at the viability results, once again a significant decrease in cell viability in F1 fresh low-motility samples is observed. As expected, reduced glutathione levels are also lower in WT cells (with high oxidative stress) than in F1 spermatozoa. In conclusion, our results could be indicating that (1) spermatozoa in this species have high levels of oxidative stress regardless of whether the males were wild or born in captivity, and these high levels of ROS could be an early indicator of susceptibility to damage but (2) in very low-quality samples, high levels of ROS are not necessarily expected because the oxidative stress process could be more advanced and specific spermatozoa damage could already be happening, producing, as an example, a direct decrease in cell viability.

However, there is a point in our study that could not be easily explained considering ROS as a factor that causes defective sperm function. When we plotted the samples, $\mathrm{DCF}^{+}$cells vs live cells considering motility ranges (Fig. 6), we observed that fast spermatozoa samples consistently appeared with high ROS levels, whereas slow spermatozoa showed low ROS levels. Although most studies in fish address the ROS issue from a negative perspective, it is intriguing how such vulnerable cells can generate these numbers of cells that are potentially dangerous for them. In mammals, it has been established that ROS play an important physiological role in spermatozoa. It is known that low levels of ROS generation are crucial for normal sperm function and are involved in important signal transduction pathways (Aitken et al. 2012). In fact, in mouse, it has been shown that sperm lipid peroxidation using a combination of ferrous ions and ascorbate increased the fertilizing potential of mouse spermatozoa by $50 \%$ (Kodama et al. 1996). Moreover, it is also known that ROS are required for compacting sperm chromatin and, paradoxically, providing protection against oxidative DNA damage (Pfeifer et al. 2001, Aitken et al. 2012). Therefore, although the underlying mechanisms of these positive effects are yet to be completely elucidated, particularly in fish, it seems clear that ROS could also be important for the regulation of sperm function.

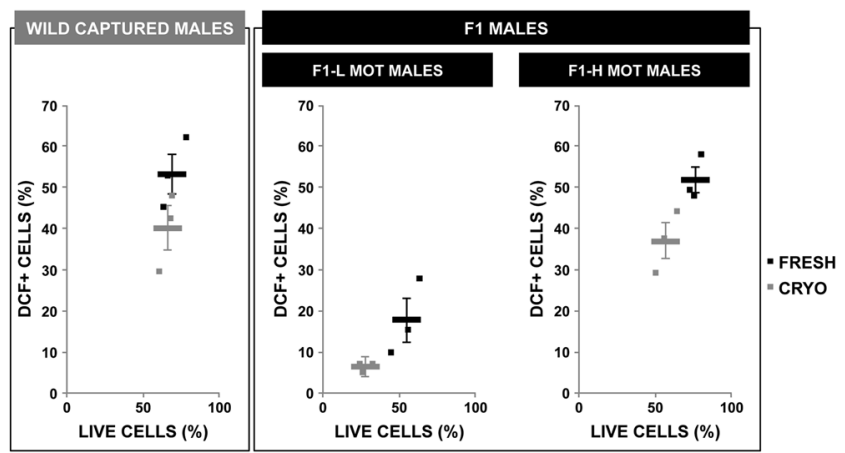

Figure 6 Viability, motility and intracellular ROS species correlation diagram. CRYO, cryopreserved/thawed samples; DCF, dichlorofluorescein; F1, F1 males; FRESH, control samples before cryopreservation; H MOT, high-motility samples; L MOT, low-motility samples; WT, wild-captured males. 
ROS localization is also another factor that matters. In our study, we observed that ROS colocalized with mitochondria, as expected, but it is also found in the nucleus, being a potential inductor of DNA damage (Fig. 5). When compared with ROS distribution in other marine flat fish (Scophthalmus maximus) spermatozoa, we observed higher levels of ROS in Solea senegalensis nuclei (Supplementary Materials 3 and 4). Whether this species has particularly high levels of oxidative stress in their spermatozoa or they are necessary for normal sperm function, we could conclude that the beneficial or detrimental effects of ROS will always be in a delicate balance and should not always be considered alarming if they are not accompanied by other negative consequences on spermatozoa.

\section{Supplementary data}

This is linked to the online version of the paper at http://dx.doi. org/10.1530/REP-16-0270.

\section{Declaration of interest}

The authors declare that there is no conflict of interest that could be perceived as prejudicing the impartiality of the research reported.

\section{Funding}

This work was financially supported by AGL2015 68330-C2-1-R project (MINECO/FEDER).

\section{Acknowledgements}

The authors would like to acknowledge AQUAGAMETE FA 1205 COST Action, Junta de Castilla y León (EDU1084/2012) and Fondo Social Europeo, Dr Olvido Chereguini for kindly providing S. maximus sperm samples for confocal microscopy, Mariano de la Hera, Planta de Cultivos el Bocal (IEO) staff, Drs Millán Cortizo and Enrique Fernández.

\section{References}

Aitken RJ \& Curry BJ 2011 Redox regulation of human sperm function: from the physiological control of sperm capacitation to the etiology of infertility and DNA damage in the germ line. Antioxidants \& Redox Signaling 14 367-381. (doi:10.1089/ars.2010.3186)

Aitken RJ, Jones KT \& Robertson SA 2012 Reactive oxygen species and sperm function - in sickness and in health. Journal of Andrology 33 1096-1106. (doi:10.2164/jandrol.112.016535)

Beirão J, Soares F, Herráez MP, Dinis MT \& Cabrita E 2009 Sperm quality evaluation in Solea senegalensis during the reproductive season at cellular level. Theriogenology 72 1251-1261. (doi:10.1016/ j.theriogenology.2009.07.021)

Beirão J, Soares F, Herráez MP, Dinis MT \& Cabrita E 2011 Changes in Solea senegalensis sperm quality throughout the year. Animal Reproduction Science 126 122-129. (doi:10.1016/j.anireprosci.2011.04.009)

Beirão J, Soares F, Pousão-Ferreira P, Diogo P, Dias J, Dinis MT, Herráez MP \& Cabrita E 2015 The effect of enriched diets on Solea senegalensis sperm quality. Aquaculture 435 187-194. (doi:10.1016/ j.aquaculture.2014.09.025)
Billard B, Cosson J, Crim LW, Suquet M 1995 Sperm physiology and quality. In Broodstock Management and Egg and Larval Quality, pp 25-52. Eds NR Bromage \& RJ Roberts. Oxford, UK: Blackwell Science.

Cabrita E, Soares F \& Dinis MT 2006 Characterization of Senegalese sole, Solea senegalensis, male broodstock in terms of sperm production and quality. Aquaculture 261 967-975. (doi:10.1016/ j.aquaculture.2006.08.020)

Cabrita E, Martínez-Páramo S, Gavaia PJ, Riesco MF, Valcarce DG, Sarasquete C, Herráez MP \& Robles V 2014 Factors enhancing fish sperm quality and emerging tools for sperm analysis. Aquaculture 432 389-401. (doi:10.1016/j.aquaculture.2014.04.034)

Chauvigné F, Boj M, Finn RN \& Cerdà J 2015 Mitochondrial aquaporin8-mediated hydrogen peroxide transport is essential for teleost spermatozoon motility. Scientific Reports 5 77-89. (doi:10.1038/ srep07789)

Dietrich GJ, Szpyrka A, Wojtczak M, Dobosz S, Goryczko K, Zakowski L \& Ciereszko A 2005 Effects of UV irradiation and hydrogen peroxide on DNA fragmentation, motility and fertilizing ability of rainbow trout (Oncorhynchus mykiss) spermatozoa. Theriogenology 64 1809-1822. (doi:10.1016/j.theriogenology.2005.04.010)

Gazo I, Shaliutina-Kolešová A, Dietrich MA, Linhartová $P$, Shaliutina $O$ \& Cosson J 2015 The effect of reactive oxygen species on motility parameters, DNA integrity, tyrosine phosphorylation and phosphatase activity of common carp (Cyprinus carpio L.) spermatozoa. Molecular Reproduction and Development 82 48-57. (doi:10.1002/ mrd.22442)

Guthrie HD \& Welch GR 2012 Effects of reactive oxygen species on sperm function. Theriogenology 78 1700-1708. (doi:10.1016/ j.theriogenology.2012.05.002)

Howell BR, Cañavate JP, Prickett R \& Conceicão LEC 2006 The cultivation of soles. Report of the 3rd workshop on the cultivation of soles. CICEM El Toruño, Cadiz, Spain, p 35.

Howell BR, Conceiçao L, Prickett R, Cañavate P \& Mañanós E 2009 Sole farming: nearly there but not quite? Aquaculture Europe 34 24-27.

Kim S-H, Yu D-H \& Kim Y-J 2010 Effects of cryopreservation on phosphatidylserine translocation, intracellular hydrogen peroxide, and DNA integrity in canine sperm. Theriogenology 73 282-292. (doi:10.1016/j.theriogenology.2009.09.011)

Kodama H, Kuribayashi Y \& Gagnon C 1996 Effect of sperm lipid peroxidation on fertilization. Journal of Andrology 17 151-157.

Koppers AJ, De Iuliis GN, Finnie JM, McLaughlin EA \& Aitken RJ 2008 Significance of mitochondrial reactive oxygen species in the generation of oxidative stress in spermatozoa. Journal of Clinical Endocrinology and Metabolism 93 3199-3207. (doi:10.1210/jc.2007-2616)

Koppers AJ, Mitchell LA, Wang P, Lin M \& Aitken RJ 2011 Phosphoinositide 3-kinase signalling pathway involvement in a truncated apoptotic cascade associated with motility loss and oxidative DNA damage in human spermatozoa. Biochemical Journal 436 687-698. (doi:10.1042/ BJ20110114)

Martínez-Páramo S, Diogo P, Beirão J, Dinis MT \& Cabrita E 2012 Sperm lipid peroxidation is correlated with differences in sperm quality during the reproductive season in precocious European sea bass (Dicentrarchus labrax) males. Aquaculture 358-359 246-252. (doi:10.1016/ j.aquaculture.2012.06.010)

Martínez-Pastor F, Cabrita E, Soares F, Anel L \& Dinis MT 2008 Multivariate cluster analysis to study motility activation of Solea senegalensis spermatozoa: a model for marine teleosts. Reproduction 135 449-459. (doi:10.1530/rep-07-0376)

Pfeifer H, Conrad M, Roethlein D, Kyriakopoulos A, Brielmeier M, Bornkamm GW \& Behne D 2001 Identification of a specific sperm nuclei selenoenzyme necessary for protamine thiol cross-linking during sperm maturation. FASEB Journal 15 1236-1238.

Porta J, Porta JM, Martínez-Rodríguez G \& Alvarez MC 2006 Genetic structure and genetic relatedness of a hatchery stock of Senegal sole (Solea senegalensis) inferred by microsatellites. Aquaculture 251 46-55. (doi:10.1016/j.aquaculture.2005.05.019)

Rasines I, Gómez M, Martín I, Rodríguez C, Mañanós E \& Chereguini O 2013 Artificial fertilisation of cultured Senegalese sole (Solea senegalensis): effects of the time of day of hormonal treatment on inducing ovulation. Aquaculture 392-395 94-97. (doi:10.1016/j. aquaculture.2013.02.011) 
Shaliutina A, Gazo I, Cosson J \& Linhart O 2013 Comparison of oxidant and antioxidant status of seminal plasma and spermatozoa of several fish species. Czech Journal of Animal Science 58 313-320.

Sieme H, Oldenhof H \& Wolkers WF 2015 Sperm membrane behaviour during cooling and cryopreservation. Reproduction in Domestic Animals 50 (Supplement 3) 20-26. (doi:10.1111/rda.12594)

Słowińska M, Nynca J, Cejko BI, Dietrich MA, Horváth Á, Urbányi B, Kotrik L \& Ciereszko A 2013 Total antioxidant capacity of fish seminal plasma. Aquaculture 400-401 101-104. (doi:10.1016/ j.aquaculture.2013.03.010)

Thomson LK, Fleming SD, Aitken RJ, De luliis GN, Zieschang J-A \& Clark AM 2009 Cryopreservation-induced human sperm DNA damage is predominantly mediated by oxidative stress rather than apoptosis. Human Reproduction 24 2061-2070. (doi:10.1093/ humrep/dep214)

Valcarce DG, Herráez MP, Chereguini O, Rodríguez C \& Robles V 2016 Selection of nonapoptotic sperm by magnetic-activated cell sorting in Senegalese sole (Solea senegalensis). Theriogenology 86 1195-1202. (doi:10.1016/j.theriogenology.2016.04.010)

Received 13 May 2016

First decision 5 June 2016

Revised manuscript received 29 July 2016

Accepted 15 August 2016 